\title{
Health care resource utilization and costs of recurrent Clostridioides difficile infection in the elderly: a real-world claims analysis
}

\author{
Winnie W Nelson, PharmD, MS, MBA; Takara A Scott, PhD; Mena Boules, MD; Christie Teigland, PhD; \\ Alexis Parente, PhD; Sudhir Unni, PhD, MBA; and Paul Feuerstadt, MD
}

\section{What is already known about this subject}

- Clostridioides difficile infection (CDI) affects more than 350,000 individuals in the United States annually and confers substantial physical, social, and emotional burden to the patient.

- The burden of recurrent CDI (rCDI) has not improved, despite a nationally decreasing trend of all CDI cases in recent years.

- Elderly individuals are at higher risk than younger individuals for CDI, rCDI, and complicated CDI.

\section{What this study adds}

- Almost $60 \%$ of patients with rCDI in the Medicare fee-for-service claims database experienced more than 1 recurrent infection.

- Among patients with rCDI, at least $20 \%$ in each rCDI cohort had 4 or more allcause inpatient admissions during the 12-month follow-up.

- Postacute care was used by $70 \%-75 \%$ of patients across rCDI cohorts.

\section{ABSTRACT}

BACKGROUND: Clostridioides difficile infection (CDI) affected an estimated 365,000 persons in the United States in 2017. Despite a nationally decreasing trend of CDI cases, the population incidence of recurrent CDI (rCDI) has not improved. Elderly individuals (aged $\geq 65$ years) are at higher risk of CDI, rCDI, and complicated CDI compared with younger individuals.

OBJECTIVE: To analyze Medicare fee-forservice data for 12 months after an initial CDI episode, in order to obtain real-world data on health care resource utilization (HRU) and costs for elderly patients with CDI and rCDI.
METHODS: A retrospective cohort study of patients who were aged $\geq 65$ years and had a first (index) CDI diagnosis from January 1 , 2010, to December 31, 2016, and continuous enrollment in Medicare Parts A, B, and D during the 12-month pre-index and 12-month post-index periods was conducted. A CDI episode was identified by either an inpatient stay with CDI diagnosis code or an outpatient medical claim with a CDI diagnosis code plus a CDI treatment. Each CDI episode was followed by a 14-day CDI claim-free period after the last CDI claim or end of CDI treatment. rCDI was a second or subsequent episode of $\mathrm{CDI}$ that occurred within an 8-week window after the 14-day CDI claim-free period. The

\author{
Author affiliations \\ Winnie W Nelson, PharmD, MS, MBA; \\ Takara A Scott, PhD; Mena Boules, MD; \\ and Sudhir Unni, PhD, MBA, Ferring \\ Pharmaceuticals, Parsippany, NJ. Christie \\ Teigland, PhD, and Alexis Parente, PhD, \\ Avalere Health, Washington, DC. Paul \\ Feuerstadt, MD, Gastroenterology Center of \\ Connecticut, Hamden, CT, and Division of \\ Gastroenterology, Yale University School of \\ Medicine, New Haven, CT.
}

AUTHOR CORRESPONDENCE: Winnie W Nelson, 973.206.4673; Winnie.nelson@ferring.com

J Manag Care Spec Pharm. 2021;27(7):828-38

Copyright $\odot 2021$, Academy of Managed Care Pharmacy. All rights reserved.

number of $C D I$ and $r C D I$ episodes, HRU, time to recurrence, and total all-cause direct medical costs were calculated over the 12-month pre-index (baseline) and 12-month follow-up periods and stratified by number of rCDI episodes (No rCDI, 1 rCDI, 2 rCDI, 3+ rCDI).

RESULTS: A total of 268,762 patients with an index CDI were included. Mean age was 78.3 years, and $69.0 \%$ were female. HRU was higher during the 6 months immediately pre-index versus 7-12 months pre-index, including a higher proportion of patients with a hospital admission ( $55.1 \%$ vs. $27.5 \%$ ) or emergency department visit ( $41.3 \%$ vs. $27.4 \%$ ), respectively. Moreover, $34.7 \%$ of the study population experienced rCDI. Of those 
who experienced 1 recurrence, $59.1 \%$ had a second recurrence, and of those who had 2 recurrences, $58.4 \%$ had a third. During the 12 -month follow-up, postacute care was used by at least $70 \%$ of each rCDI cohort. The proportion of patients with $\geq 4$ hospital admissions during follow-up was highest for the $3+$ rCDI cohort ( $24.9 \%$ of patients). During the 12-month follow-up, mean total all-cause direct costs were $\$ 76,024, \$ 99,348, \$ 96,148$, and $\$ 96,517$ for the No rCDI, 1 rCDI, 2 rCDI, and 3+rCDI cohorts, respectively, largely driven by inpatient costs. Adjusted all-cause total costs were significantly higher for all 3 rCDI cohorts compared with the No rCDI cohort.

CONCLUSIONS: Elderly individuals experienced high rates of recurrence after their first CDI episode, and especially after a prior recurrence. The intensity of HRU during follow-up was higher for patients who suffered recurrences. Patients with rCDI had the burden of higher costs of care, including the patient out-of-pocket responsibility, versus patients with a single CDI episode.

Clostridioides difficile infection (CDI) affected an estimated 365,000 persons in the United States in 2017 and is recognized as the most common health care-associated infection in the United States. ${ }^{1-3}$ Although rates of health care-associated CDI cases and CDI-related mortality have decreased over the past decade, rates of recurrence and communityassociated CDI cases remain stagnant., ${ }^{2,4}$

Patients with CDI experience diarrhea that can vary from mild to severe, possible abdominal cramping, and significant physical discomfort and social withdrawal. ${ }^{5,6}$ Approximately $25 \%$ of all patients with a first CDI episode experience recurrent CDI (rCDI), and approximately $50 \%$ of patients who develop rCDI go on to endure another recurrence..$^{7-9}$ Direct medical costs related to rCDI in the United States are estimated at $\$ 2.8$ billion annually, with higher per-patient costs associated with CDI-related surgery or hospitalization during the year after rCDI, as well as a higher likelihood of death. ${ }^{10}$

There appears to be an increase in CDI cases that are refractory to conventional therapies, with 1 study showing a $188.8 \%$ increase in multiple rCDI events between 2001 and 2012. ${ }^{1}$ The burden of CDI recurrences, as reported by population incidence, has not improved despite a nationally decreasing trend of CDI cases between 2011 and 2017. ${ }^{2}$ This persistence of refractory disease underscores the importance of reducing rCDI. Despite the success of infection prevention practices, antimicrobial stewardship, and more aggressive therapies in decreasing health care-associated $\mathrm{CDI}$, the rate of rCDI has not improved and has been shown to be a more intractable problem.
Although several modifiable and nonmodifiable risk factors for CDI exist, advanced age (aged $\geq 65$ years), which may serve as a proxy for comorbidities and disease severity, is considered one of the most important nonmodifiable risk factors for CDI and rCDI.1-13 Elderly individuals experience $\mathrm{CDI}$ at a much higher rate than younger persons, with an estimated incidence of $\sim 500$ cases per 100,000 persons, compared with $\sim 90$ cases per 100,000 persons among all adults in the United States. ${ }^{14,15}$ The elderly are not only at increased risk for initial infection and recurrence but are also 3 times more likely to develop complicated CDI compared with a younger cohort. ${ }^{11}$ Older age is also a known risk factor for rCDI, with adults aged $\geq 65$ years having a $63 \%$ higher risk of recurrence than younger persons, with each additional year of age increasing the risk. ${ }^{13,16}$

There are limited real-world outcome data considering health care resource utilization (HRU) and costs for elderly patients with CDI and rCDI. We, therefore, analyzed Medicare fee-for-service (FFS) data to describe 12-month recurrence rates, health care resource utilization, and total costs in patients with a single episode of CDI and those with recurrent CDI.

\section{Methods}

\section{STUDY DESIGN}

A retrospective cohort study was conducted to assess HRU and cost outcomes for patients with CDI and rCDI using data from the $100 \%$ Medicare FFS claims database, which contains inpatient, outpatient, demographic, and enrollment information, and the 100\% Prescription Drug Event dataset, which includes prescription drug claims for Medicare Part D.

The 100\% Medicare FFS data were accessed by Avalere through their parent company Inovalon, which has been designated by the Centers for Medicare and Medicaid Services (CMS) as a qualified entity, allowing research to be conducted under a Health Insurance Portability and Accountability Act (HIPAA)-compliant, research-focused data-use agreement. Specifically, the use of data in this study abided by the HIPAA requirements for the privacy and security of protected health information.

\section{DATA SOURCE}

The Medicare FFS claims database contains health service utilization data for Medicare beneficiaries with traditional Medicare insurance coverage whose providers were paid directly by CMS. The data do not include Medicare beneficiaries covered by managed care organizations (Medicare Advantage). ${ }^{17}$ During the study timeframe of January 1, 2009, 
through December 31, 2017, 67\%-77\% of Medicare beneficiaries received FFS benefits, and the remainder received Medicare benefits through a Medicare Advantage plan. ${ }^{18}$ The FFS data are derived from reimbursement information generated through billing processes from health care providers and includes Medicare enrollment data from CMS describing coverage dates and demographic characteristics of beneficiaries. The claims database contains information about covered health care services used by Medicare beneficiaries, including date of service; diagnoses; procedures performed; place of service; provider information; CMS and beneficiary paid amounts; and demographic data, such as age, sex, dual-eligible status for Medicaid, reason for entitlement to Medicare (aged 65 years or disabled aged $<65$ years), and race/ethnicity.

Data from Medicare Part A contain diagnosis and billing information for inpatient care received while in a hospital or postacute care (PAC) setting (e.g., skilled nursing facility, home health agency services, inpatient rehabilitation facility or long-term acute care hospital).$^{19}$ The data do not contain service details in the inpatient setting, such as medication use. Data from Medicare Part B pertain to information for outpatient medical care including physician office visits, lab tests and procedures performed in outpatient clinics, and other services such as durable medical equipment and mental health care..$^{20}$ Data from Medicare Part D cover reimbursement for outpatient prescription drugs. ${ }^{21}$ Medicare prescription drug coverage is an optional benefit offered to beneficiaries, and they may access the benefits via a Medicare prescription drug plan. Not available in the claims data are medications used during an inpatient stay, as the information is bundled within the diagnosis-related group payment. Prescription or over-the-counter medications that were paid $100 \%$ out of pocket by the patients are also not observable.

\section{PATIENT IDENTIFICATION}

Claims were analyzed for patients who were aged $\geq 65$ years and had a first (index) CDI diagnosis; all included patients must have had continuous enrollment in Medicare Parts A, $\mathrm{B}$, and $\mathrm{D}$ during the 12 -month pre-index and 12-month postindex observation periods. An episode of CDI was identified based on either an inpatient stay attributed to a CDI diagnosis code (Supplementary Table 1, available in online article) or an outpatient medical claim with a CDI diagnosis code plus confirmation of an appropriate CDI treatment. Acceptable CDI treatments included vancomycin, fidaxomicin, metronidazole, rifaximin, bezlotoxumab, or fecal microbiota transplant (Supplementary Table 1). The first date for index CDI identification was January 1, 2010, and the last observation date was December 31, 2016, to account for the 12-month pre- and post-index follow-up periods. During the 12-month pre-index period, by study definition, there were no CDI-related claims.

To standardize the start date of events across cases, a CDI episode began on the date of the first CDI medical claim and included consecutive medical and prescription drug claims with a diagnosis of CDI. To be considered part of the episode, each CDI claim needed to occur within 14 days or less from the previous CDI claim, with multiple CDI claims composing a CDI episode. Each CDI episode was followed by a 14-day CDI claim-free period following the last CDI claim or end of CDI treatment, to increase the likelihood that any subsequent CDI claims were associated with a distinct CDI episode and not with the same episode of CDI (Supplementary Figure 1a, available in online article). An episode of rCDI was defined as a second or subsequent episode of CDI, using the same criteria for the index CDI episode, that occurred within an 8-week window following the 14-day CDI claim-free period (Supplementary Figure 1b, available in online article). Multiple rCDI episodes were analyzed up to 12 months following the index CDI date. CDI events that occurred later than each 8-week window were not counted as rCDI (in accordance with rCDI definition from the U.S. Centers for Disease Control and Prevention ${ }^{22}$ ), but as new infections, and were not included in this analysis to avoid misclassification.

Deidentified demographic and clinical characteristics were evaluated for each individual, including age; sex; geographic region; race/ethnicity; Charlson Comorbidity Index (CCI); comorbid conditions; outpatient medication exposure (any use of gastric acid-suppressing agents, antimicrobials, or immunosuppressant agents); pre- and post-index medical procedures and treatments (transplants, gastrointestinal surgery, enteral feeding, chemotherapy); HRU; and costs.

The 12-month pre-index period was separately reported as $0-6$ months pre-index (i.e., the 6 months immediately before the index CDI) and 7-12 months pre-index. The 0 - to 6-month pre-index period captured potential precipitating events of CDI, whereas the 7- to 12-month pre-index period represented the baseline health characteristics of the cohorts before the occurrence of clinical events that likely predisposed the patient to their index CDI episode. To serve as a benchmark reference, select baseline and demographic data for the national Medicare population were extracted for all beneficiaries who were enrolled in Medicare FFS with medical and pharmacy benefits for at least 6 months between January 1, 2016, and December 31, 2016. 


\section{OUTCOMES}

The number of CDI and rCDI episodes; HRU (inpatient admissions, intensive care unit stays, outpatient visits including emergency department [ED] visits, and PAC); time to recurrence; and total all-cause direct costs (medical and pharmacy costs, by Medicare and patient portions) were calculated over the 12-month pre-index (baseline) period and 12-month follow-up periods and stratified by number of rCDI episodes (No rCDI, 1 rCDI, 2 rCDI, or 3+ rCDI).

\section{DATA ANALYSIS}

Descriptive analyses of the demographic characteristics, costs, and HRU data were conducted for this study. For categorical variables, counts and percentages were used; for continuous variables, measures of central tendency (mean [SD]) were used. Costs were adjusted to 2018 dollar values using the medical care component of the Consumer Price Index. ${ }^{23}$

Multivariate regression modeling was used to evaluate differences in total all-cause costs between the No rCDI cohort (reference group) and each of the recurrence cohorts. Specifically, we estimated a generalized linear model using gamma distribution with log-link function. The covariates were baseline characteristics including demographics (age, gender, and census region); comorbidities (CCI score, chronic pulmonary disease, cardiovascular disease, diabetes, heart failure, peripheral vascular disease, and renal disease); medication exposure (antibiotics, chemotherapy, and gastric acid-suppressing agents); and procedures (gastrointestinal surgery and transplant).

All analyses were conducted with SAS software package version 9.4 (SAS Institute, Cary, NC, USA).

\section{Results}

\section{DEMOGRAPHICS}

A total of 268,762 patients with an index CDI were identified and included in the study (Table 1): $65.3 \%$ had No rCDI, $14.2 \%$ experienced $1 \mathrm{rCDI}, 8.5 \%$ had 2 episodes of rCDI, and $12.0 \%$ had $3+$ rCDI. For all patients with CDI, the mean age was 78.3 years, $69.0 \%$ were female, and the mean (SD) CCI was 5.2 (3.4). For reference, in the national Medicare cohort of 4.8 million beneficiaries (including the CDI subgroup of $269 \mathrm{k}$ patients reported here), mean age was 75.4 years, $59.1 \%$ were female, and the mean (SD) CCI was 4.0 (3.8). The most common geographic regions of patients with CDI were the South (36.7\%) and Midwest (25.8\%), similar to the national Medicare cohort.

\section{PRE-INDEX PERIOD}

During the 12 months immediately preceding the index CDI episode, more than $83 \%(145,743$ of 175,554$)$ of patients in the No rCDI cohort received outpatient antimicrobials, and more than half received outpatient prescriptions for gastric acid-suppressing medications; antimicrobial use was higher in cohorts with more rCDI episodes (Table 1). There was a similar trend for proportion of patients receiving pre-index chemotherapy or transplants (solid organ or bone marrow). The proportion of patients with pre-index transplants in the $3+\mathrm{rCDI}$ cohort was 4 times higher than in the $2 \mathrm{rCDI}$ cohort and 9 times higher than the No rCDI cohort.

The two 6-month pre-index periods reflected distinctly different levels of HRU. HRU was higher during the 6 months immediately pre-index compared with 7-12 months before the index CDI episode. Comparing the 0- to 6-month preindex period with the 7- to 12-month pre-index period, the proportion of patients was 2 times higher with $\geq 1$ hospital admission ( $55.1 \%$ vs. $27.5 \%), \geq 1 \mathrm{ED}$ visit ( $41.3 \%$ vs. $27.4 \%$ ), and $\geq 4$ outpatient visits $(86.2 \%$ vs. $78.0 \%)$ just before the index CDI. In the 0 - to 6 -month pre-index period, the highest rates of inpatient admission and ED visit occurred in the 3+ rCDI cohort (Table 1).

\section{POST-INDEX PERIOD}

Based on the patient counts in the mutually exclusive study cohorts, the rates of recurrence among patients with or without previous recurrence can be observed. Of the study population of 268,763 patients, 93,208 (34.7\%) had 1 or more recurrence. The rate of subsequent rCDI was higher in patients with a previous recurrence. Of those who experienced 1 recurrence, $59.1 \%$ had a second recurrence $(55,045$ of 93,208 patients), and of those who had 2 recurrences, $58.4 \%$ had a third $(32,147$ of 55,045 patients).

The mean (SD) time to first recurrence ranged from 25 (10) days in patients with 3+ recurrences to 34 (16) days in patients with $1 \mathrm{rCDI}$ (Figure 1). Relative to the time to first recurrence, there was a longer time (33.0 [13.0] days to 44.0 [14.7] days) to subsequent recurrent episode(s) after the first recurrence in patients with multiple rCDI.

During the 12-month follow-up, HRU varied by number of recurrences (Table 2). There was a trend for increased hospital length of stay (LOS) among patients with any recurrence (17.6-17.9 days) compared with patients with no recurrence (13.4 days). Patients with recurrences had numerically higher rates of ED visits and inpatient admissions compared with those without recurrent disease. Patients with 3+ rCDI had an average of 2.5 hospital admissions and 1.6 ED visits during the 12-month follow-up. Postacute care was used by at least $70 \%$ of each rCDI cohort during follow-up. The proportion of patients with $\geq 4$ hospital admissions during 


\section{TABLE 1 Patient Characteristics, CDI-Specific Risk Factors, and Health Care Resource Utilization During 12-Month Pre-Index Period}

\begin{tabular}{|c|c|c|c|c|c|}
\hline & $\begin{array}{c}\text { No rCDI } \\
(n=175,554)\end{array}$ & $\begin{array}{c}1 \text { rCDI } \\
(n=38,163)\end{array}$ & $\begin{array}{c}2 \text { rCDI } \\
(n=22,898)\end{array}$ & $\begin{array}{c}3+\text { rCDI } \\
(n=32,147)\end{array}$ & $\begin{array}{l}\text { National Cohort } \\
(n=4,797,475)\end{array}$ \\
\hline \multicolumn{6}{|l|}{ Demographics } \\
\hline Age, mean (SD), years & $78.5(8.0)$ & $78.1(7.9)$ & $78.3(7.9)$ & $77.9(8.0)$ & $75.4(7.9)$ \\
\hline Female, \% & 69.2 & 69.1 & 69.0 & 67.5 & 59.1 \\
\hline \multicolumn{6}{|l|}{ Census region, $\%$} \\
\hline Northeast & 21.0 & 21.8 & 21.8 & 22.2 & 19.3 \\
\hline Midwest & 25.1 & 26.0 & 26.7 & 28.8 & 23.1 \\
\hline South & 37.7 & 36.5 & 35.7 & 32.5 & 38.4 \\
\hline West & 16.0 & 15.6 & 15.8 & 16.4 & 18.9 \\
\hline Unknown & 0.2 & 0.1 & 0.1 & 0.04 & 0.3 \\
\hline \multicolumn{6}{|l|}{ Medical comorbidities } \\
\hline CCI score, mean (SD) & $5.0(3.4)$ & $5.2(3.4)$ & $5.2(3.4)$ & $5.2(3.5)$ & $4.0(3.8)$ \\
\hline Chronic pulmonary disease, \% & 49.5 & 50.6 & 50.5 & 49.4 & 40.7 \\
\hline CVD, \% & 38.3 & 39.9 & 39.1 & 37.3 & 33.3 \\
\hline Diabetes, \% & 45.5 & 45.8 & 45.2 & 44.3 & 41.7 \\
\hline Heart failure, \% & 43.3 & 45.3 & 44.5 & 43.5 & 24.0 \\
\hline PVD, \% & 42.8 & 44.6 & 44.1 & 43.0 & 35.4 \\
\hline Renal disease, \% & 37.6 & 39.7 & 40.4 & 43.2 & 22.6 \\
\hline \multicolumn{6}{|l|}{ Outpatient medication exposure, \% } \\
\hline Antimicrobials & 83.0 & 85.3 & 86.8 & 89.8 & - \\
\hline Chemotherapy & 44.4 & 45.8 & 46.0 & 50.0 & - \\
\hline Gastric acid-suppressing agents & 50.6 & 51.1 & 52.1 & 54.0 & - \\
\hline \multicolumn{6}{|l|}{ Procedures, \% } \\
\hline GI surgery & 3.9 & 4.5 & 4.2 & 4.3 & - \\
\hline Transplant & 1.4 & 2.3 & 3.4 & 12.4 & - \\
\hline
\end{tabular}

\section{Health care resource utilization}

0-6 months pre-index

Patients with inpatient admission, \%

Patients with ED visit, \%

Number of outpatient visits per patient, mean (SD)

\begin{tabular}{|c|c|c|c|c}
\hline 52.8 & 58.0 & 59.4 & 60.7 & - \\
\hline 40.2 & 41.6 & 43.7 & 44.8 & - \\
\hline $10.4(7.9)$ & $11.2(8.4)$ & $11.5(8.5)$ & $12.4(9.1)$ & - \\
\hline
\end{tabular}

7-12 months pre-index

\begin{tabular}{l|c|c|c|c}
\hline Patients with inpatient admission, \% & 27.4 & 27.7 & 27.5 & 27.7 \\
\hline Patients with ED visit, \% & 27.1 & 27.5 & 28.3 & 27.8 \\
\hline $\begin{array}{l}\text { Number of outpatient visits per patient, } \\
\text { mean (SD) }\end{array}$ & $8.8(7.3)$ & $9.2(7.6)$ & $9.2(7.5)$ & - \\
\hline
\end{tabular}

a Select reference data were obtained from the entire population of patients who were enrolled in Medicare FFS with medical and pharmacy benefits for at least 6 months between January 1, 2016, and December 31, 2016.

$\mathrm{CCl}=$ Charlson Comorbidity Index; $\mathrm{CDI}=$ Clostridioides difficile infection; $\mathrm{CVD}=$ cerebrovascular disease; $E D=$ emergency department; FFS=fee-for-service; $\mathrm{GI}$ = gastrointestinal; $P V D=$ peripheral vascular disease; $r C D I=$ recurrent Clostridioides difficile infection. 


\section{FIGURE 1 Mean Time to CDI Recurrence, by rCDI Cohort and Recurrence Episode}
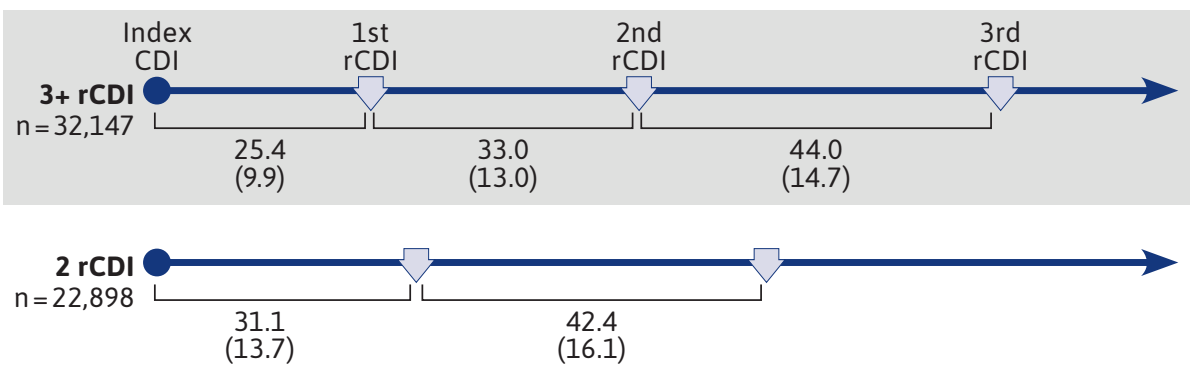

1 rCDI $n=38,163$

Note: Mean time to recurrence is approximately 1 month, with a longer time to subsequent rCDI episodes in patients with multiple recurrences. Data are presented as mean (SD) days.

$C D I=$ Clostridioides difficile infection; $r C D I=$ recurrent Clostridioides difficile infection.

follow-up was $12.8 \%$ for patients with No rCDI and higher for cohorts with more rCDI episodes (19.9\%, 22.1\%, and $24.9 \%$, respectively; Figure 2).

During the 12-month follow-up, mean total all-cause direct costs (Medicare and patient payments) were $\$ 76,024, \$ 99,348, \$ 96,148$, and $\$ 96,517$ for No rCDI, 1 rCDI, 2 rCDI, and 3+ rCDI cohorts, respectively, indicating that patients who suffered rCDI incurred higher costs. These costs were reported as totals, as well as divided into the portion paid by Medicare and the patient's copayment (Table 3 ). When observed by types of health care services, total all-cause medical costs were driven largely by inpatient costs. Prescription costs comprised $7.2 \%-10.3 \%$ of total costs in the rCDI cohorts. Costs for the $3+$ rCDI cohort were elevated relative to the No rCDI cohort: $38 \%$ more inpatient spending, 41\% more outpatient spending, 40\% more PAC spending, and 50\% more prescription spending. The patient portion of total costs was greater for patients with any recurrence than for patients with no recurrence: by increasing rCDI cohort, $\$ 9,621$, $\$ 12,089, \$ 11,690$, and $\$ 11,392$. In the adjusted all cause costs analysis, after controlling for baseline demographic and clinical characteristics, patients in the $1 \mathrm{rCDI}, 2$ rCDI, and $3+$ rCDI subgroups had significantly higher overall 12-month costs than those in the No rCDI subgroup, with adjusted differences of $\$ 21,413, \$ 16,282$, and $\$ 14,433$, respectively $(\mathrm{P}<0.0001)$.

\section{Discussion}

Recurrence is a major problem for patients suffering from C. difficile infection. In this population of Medicare patients aged 65 years and older, a substantial proportion of patients who had CDI experienced a recurrence: nearly $35 \%$ of the patients with an index episode had $\geq 1$ recurrence within 12 months, and 59\% of patients who had 1 recurrence had another episode. These rates are in the upper range of what has been reported previously in the literature, potentially reflecting data in this study from a broader range of health care settings as well as an older (Medicare beneficiaries aged $\geq 65$ years) population. ${ }^{7-9}$ The time to recurrence across all 3 rCDI cohorts was approximately 1 month in our study, similar to data reported elsewhere. ${ }^{24,25}$ This 4-week time period presents a known, but often not actioned, window of opportunity to modify underlying risk factors and reduce the risks for recurrence.

The characteristics of the CDI cohort in the current study were validated when compared with previous research. Viewed alongside the national Medicare population, the selected characteristics of patients with CDI showed a constellation of factors consistent with those who are known to be more susceptible to CDI. Patients with CDI were older on average, and chronic conditions, such as congestive heart failure, renal disease, and chronic pulmonary disease, were more common. Similar trends were seen in another study of Medicare beneficiaries with CDI. ${ }^{26}$ Usage of outpatient antimicrobial or acid-suppressive therapy was high before the index episode of CDI; this is consistent with the elderly population generally having higher use of these therapies. Similar to what was seen in this study, previous studies in the United States showed that the highest adjusted rates of CDI were recorded in the South, mirroring the geographic distribution of the national Medicare cohort. Geographic variance in CDI rates has been partially attributed to variability in patient age and CDI testing rates by region. ${ }^{12,27}$ Therefore, our study is consistent with previous analyses considering these baseline factors. As the medication data were derived from only Medicare Part D covered prescription claims, this should only be used as contextual information. 


\begin{tabular}{|c|c|c|c|c|}
\hline & No $r C D I(n=175,554)$ & 1 rCDI $(n=38,163)$ & 2 rCDI $(n=22,898)$ & $3+r C D I(n=32,147)$ \\
\hline Patients with inpatient admission, $\%$ & 85.1 & 87.7 & 87.0 & 86.5 \\
\hline Inpatient admissions per patient, mean (SD) & $1.8(1.7)$ & $2.3(1.9)$ & $2.4(2.0)$ & $2.5(2.1)$ \\
\hline LOS per admission, mean (SD), days & $13.4(17.2)$ & $17.9(20.6)$ & $17.6(20.3)$ & $17.6(21.2)$ \\
\hline Patients with ED visit, \% & 50.2 & 54.6 & 55.4 & 57.9 \\
\hline ED visits per patient, mean (SD) & $1.3(2.3)$ & $1.4(2.3)$ & $1.5(2.7)$ & $1.6(2.8)$ \\
\hline Outpatient visits per patient, mean (SD) & $22.9(16.2)$ & $25.2(17.1)$ & $24.8(16.7)$ & $26.3(17.4)$ \\
\hline Patients with postacute care, ${ }^{a} \%$ & 69.9 & 74.6 & 72.2 & 69.8 \\
\hline LTACH & 5.1 & 10.8 & 8.4 & 5.3 \\
\hline IRF & 7.0 & 10.9 & 8.8 & 8.3 \\
\hline SNF & 44.7 & 49.4 & 45.1 & 40.0 \\
\hline $\mathrm{HHA}$ & 50.1 & 54.7 & 54.8 & 53.6 \\
\hline \multicolumn{5}{|c|}{$\begin{array}{l}\text { aPostacute care was defined as a stay in a skilled nursing facility, inpatient rehabilitation facility, or long-term acute care hospital or services provided by a home } \\
\text { health agency. Patients could have had treatment in } 1 \text { or more PAC settings after index CDI and, therefore, the subgroup numbers may not add to } 100 \% \text {. }\end{array}$} \\
\hline \multicolumn{5}{|c|}{$\begin{array}{l}C D I=C \text { lostridioides difficile infection; } E D=\text { emergency department; } H H A=\text { home health agency; } I R F=\text { inpatient rehabilitation facility; } L O S=\text { length of stay; } \\
L T A C H=\text { long-term acute care hospital; } P A C=\text { postacute care; } r C D I=\text { recurrent Clostridioides difficile infection; } S N F=\text { skilled nursing facility. }\end{array}$} \\
\hline
\end{tabular}

The Medicare FFS dataset allows for longer study periods because, unlike younger populations with employer-provided health insurance, Medicare beneficiaries usually remain with Medicare coverage for life and do not change insurance to alternative Medicare Advantage plans frequently. This longer window provided the opportunity to observe the pre-index period immediately before the index CDI as well as another 6 months earlier (7-12 months before the index CDI). The differences in the level of health care encounters between these 2 periods were stark, with a much higher rate of utilization in the 6 months immediately before the index CDI event, compared with 7-12 months before the index CDI. The differences were particularly notable for inpatient admissions and ED visits. Recent health care exposure, especially an inpatient stay, is a known risk factor for CDI. ${ }^{16,28,29}$ The increased likelihood of experiencing CDI from health care exposure may be due to the presence of more medical comorbidities that require frequent health care visits, severe underlying disease that weakens the immune system, or exposure to patients with active CDI (especially during an inpatient stay). Conversely, the level of health care encounters during the 7-12 months before the index CDI episode likely represented the baseline health of the population, which was similar across the study cohorts.

In addition to the heavy physical burden of experiencing $\mathrm{CDI}$, patients also have a substantial burden of health care utilization after CDI. After the initial episode of CDI, all
rCDI cohorts had high rates of all-cause hospitalizations, ED visits, and outpatient visits during the 12-month followup. Over half of the patients with CDI had an ED visit, and at least $85 \%$ of patients in each cohort had an inpatient admission following their index presentation. In contrast, during the 7- to 12-month pre-index baseline period, $27 \%$ of these patients had an inpatient admission. Furthermore, the intensity of inpatient care was higher for patients who suffered recurrences. Between $20 \%$ and $25 \%$ of patients with $\mathrm{rCDI}$ had 4 or more admissions during the 12-month follow-up (13\% among those with No rCDI). Higher inpatient care intensity among rCDI cohorts was also seen in terms of hospital LOS. The average LOS per inpatient admission was between 17.6 and 17.9 days among patients with rCDI, compared with 13.4 days among those with no recurrence in our study, that is, an average increase of more than 4 days (>30\% longer LOS). Compared with LOS data from the literature, our findings show a much longer duration. A survey of the State Inpatient Databases reported a LOS of 7.3 days for a primary rCDI diagnosis in U.S. adults. ${ }^{30}$ Excess LOS attributable to CDI is approximately 3 days..$^{31} \mathrm{~A}$ previous analysis of Medicare claims data for patients with community-acquired CDI showed a median hospital LOS of 5 days (interquartile range, $3-8$ days). ${ }^{32}$ Our study included all cases of CDI and rCDI (community associated and health care associated) in a population aged $\geq 65$ years and, thus, provided a more comprehensive and age group-specific reporting of inpatient resource use. 


\section{FIGURE 2 Hospital Admissions During 12-Month Follow-up Period}

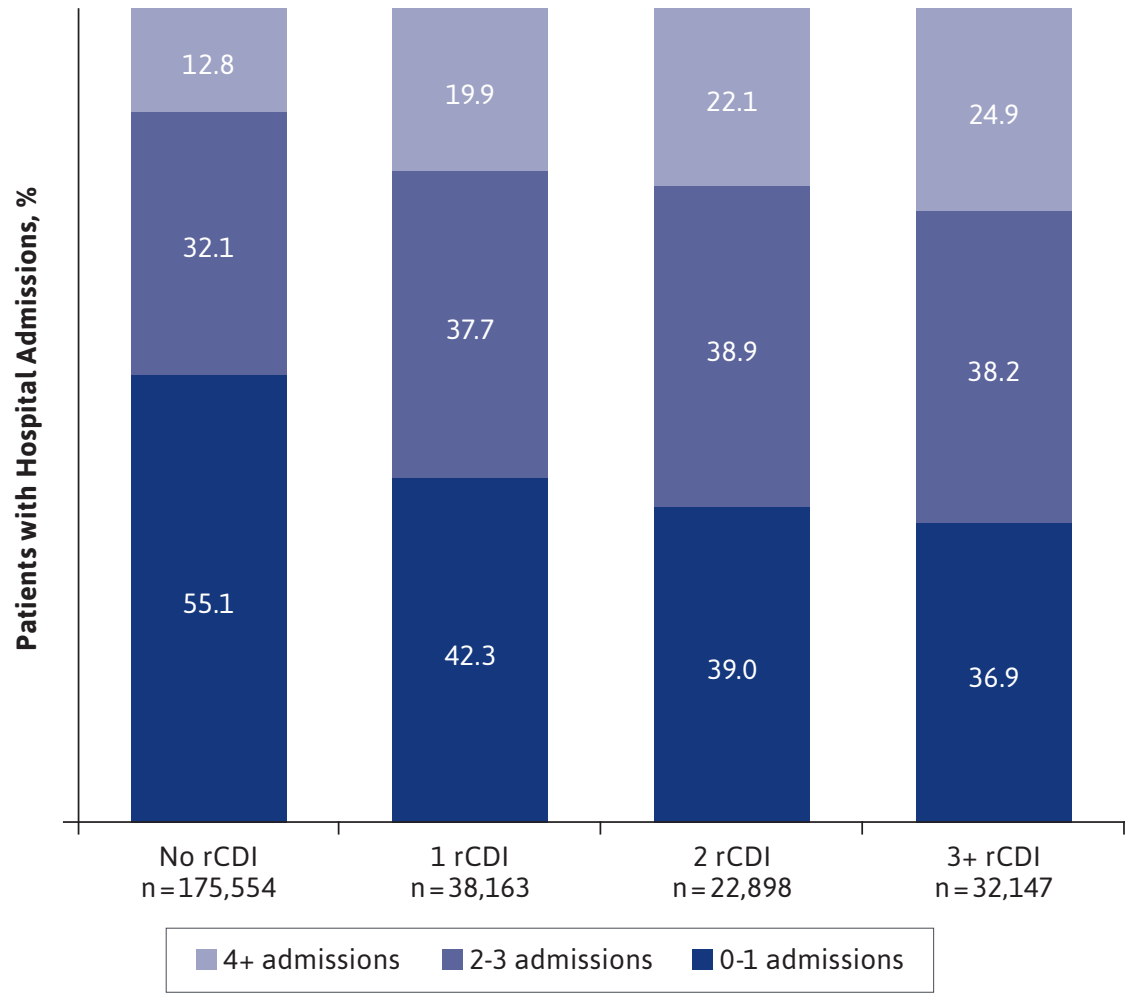

Note: Rates of $\geq 4$ inpatient admissions were higher with greater number of $r C D I$ episodes. $\mathrm{rCDI}=$ recurrent Clostridioides difficile infection.

The Medicare dataset provided specific details regarding PAC and further separated the care setting into home health, skilled nursing facility, inpatient rehabilitation facility, or long-term acute care hospital. In 2017, approximately $10.8 \%$ of all Medicare beneficiaries used some form of PAC. ${ }^{33}$ In comparison, a much higher proportion of our study population, between $70 \%$ and $75 \%$, used PAC during the 12-month follow-up. The PAC setting provides necessary, goal-oriented care for an elderly patient in a structured setting. Patients with health careassociated CDI who are discharged to a skilled nursing facility have a much lower risk of readmission within 30 days than patients who have a routine discharge into the community, indicating that PAC can be beneficial in the management of CDI in older patients..$^{34}$ On the other hand, these facilities are also associated with a higher risk of transmission of CDI, with an incidence of approximately 2.3 CDI cases $/ 10,000$ resident-days, which can vary by PAC facility type and specific institution. ${ }^{35,36}$ Therefore, the use of PAC has the potential for both positive and negative effect on the health care experience of patients with CDI or rCDI. Despite CMS limits on PAC benefits, the use among our study population was exceptionally high. Future research on the effect of different types of PAC use in CDI is warranted.
Experiencing CDI is expensive. In our study, patients covered by the Medicare FFS program who suffered CDI incurred substantial total all-cause medical, pharmacy, and patient-paid costs in the year after a first CDI episode, and the patients were responsible for $12 \%$ of the payment on average, despite Medicare coverage. As challenging as rCDI is from a quality-of-life standpoint, patients with rCDI have the additional burden of a higher cost of care, compared with patients with a single CDI episode. These higher total costs for rCDI were largely driven by higher rates of hospitalizations, multiple hospitalizations, greater utilization of outpatient services, and higher Medicare Part D covered prescription drug costs. Our study is consistent with previous analyses showing higher costs for those with any rCDI compared with those with no recurrence. ${ }^{24,37}$ However, we observed similar total costs no matter the recurrence cohort in the follow-up period. This was likely attributed to lower utilization of typically costly PAC services and higher costs for inpatient, outpatient, physician services, and drug usage in the cohorts with 2 or more recurrences. rCDI is both physically and financially challenging for patients and, because it disproportionately affects the elderly, it is imperative to ensure that medical care is affordable for this group. Advances in therapeutic options to reduce rCDI can potentially play a role in easing both the cost and physical burden among elderly patients.

\section{LIMITATIONS}

This study has some limitations. Our study was designed to evaluate realworld burden of CDI and rCDI, with the study objective of reporting economic data for a full 12-month period. We, 


\section{TABLE 3 Mean Total All-Cause Direct Costs per Patient During 12-Month Follow-up}

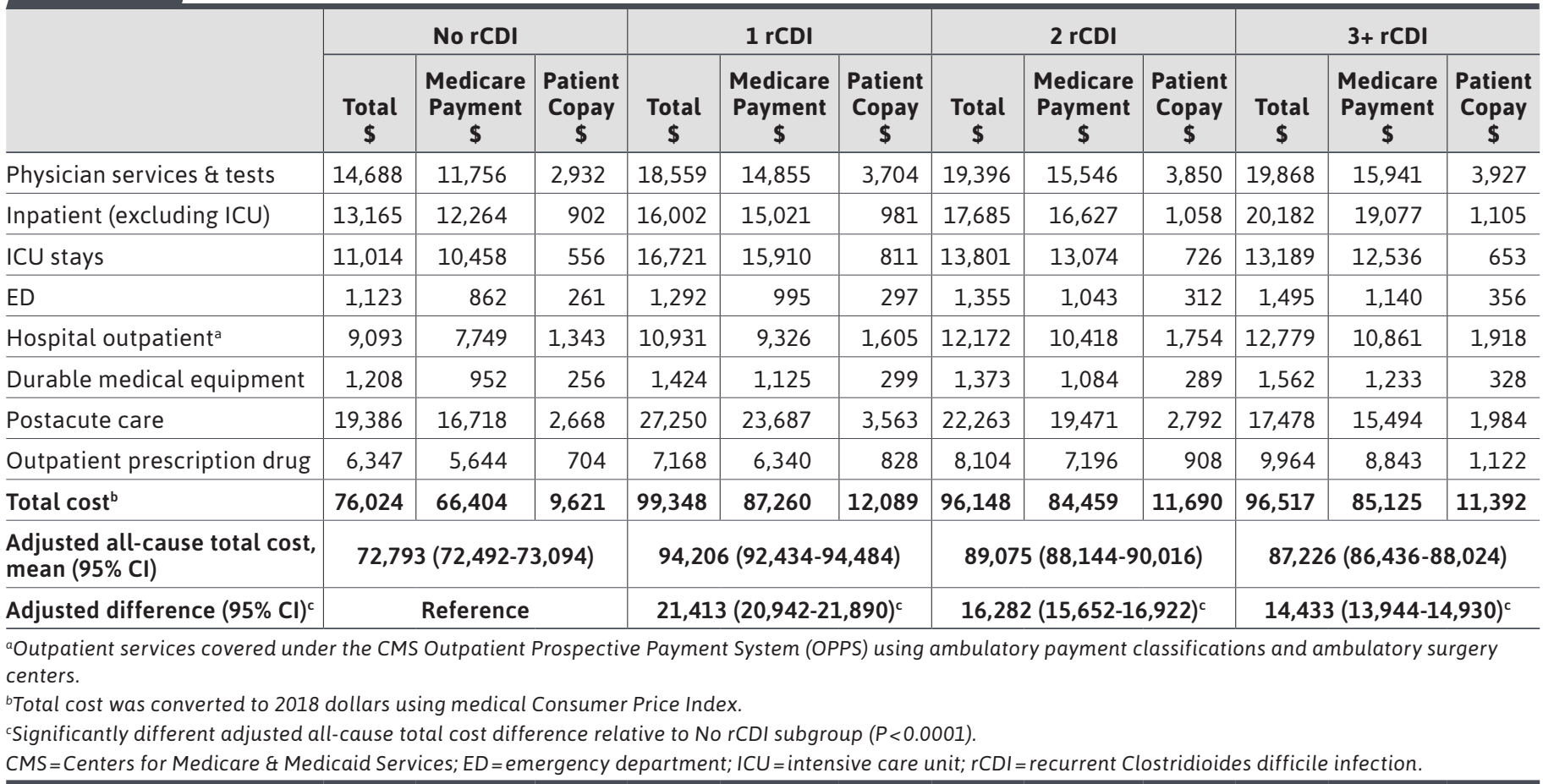

therefore, were unable to include patients who died within 12 months following their index CDI episode. We understand that elderly patients with CDI suffer higher rates of mortality than younger patients, ${ }^{11,32,38}$ but the current study design avoided the distortion of the economic burden caused by the end-of-life experience. On balance, the analysis fulfills the objective of providing a fair representation of economic burden of $\mathrm{CDI}$ and $\mathrm{rCDI}$ in the elderly population.

Another limitation of the study is its use of the Medicare FFS program, which may not reflect the experience of patients with CDI who are covered by other benefit programs, such as those enrolled in privately managed Medicare Advantage plans. However, many similar data trends were seen in a real-world analysis of U.S. commercial claims for patients with CDI, including time to recurrence of approximately 1 month, follow-up costs being higher for rCDI cohorts (with inpatient costs representing the majority of total costs), and rates of HRU being highest for the $3+$ rCDI cohort. ${ }^{24}$ The coverage policy of Medicare is more stringent than that of commercial insurance, and, therefore, a range of services not covered by Medicare could have been provided and paid for by the patient or supplemental insurance. Providers do not submit claims for these services to CMS, and the data were therefore not accounted for in our analysis. As a claims-based analysis, there may be random misclassifications or missing diagnoses of CDI events.

Nevertheless, we believe that our study contributes significantly to the literature on CDI trends because it reports on a large, representative sample of elderly patients with CDI and rCDI in the United States from 100\% Medicare FFS claims data that includes HRU and expenditures across all settings of patient care from a payer perspective. The use of all-cause costs as an outcome measure provides a complete picture of cost burden for population decision makers and is supported by current methodologic recommendations. ${ }^{39,40}$ In addition, the use of disease codes and drug codes is not sufficiently reliable to separate disease-related and disease-unrelated costs.

\section{Conclusions}

CDI and rCDI are associated with substantial health care costs and resource utilization in the Medicare population. Reduction of recurrent episodes is essential to reduce the economic burden of CDI. 


\section{DISCLOSURES}

Funding for this study was provided by Ferring Pharmaceuticals. Nelson is an employee of Ferring Pharmaceuticals, and Scott, Boules, and Unni were employees of Ferring Pharmaceuticals at the time of this study. Teigland and Parente are employees of Avalere Health and provided consulting services to Ferring Pharmaceuticals. Feuerstadt has served as a consultant to and on the speakers bureau for Merck and Co. and has served as a consultant for Ferring Pharmaceuticals and Roche Pharmaceuticals.

Portions of the data contained in this study appeared as an abstract/ePoster for the AMCP Annual Meeting 2020, April 2020

\section{ACKNOWLEDGMENTS}

Medical writing and editorial support were provided by Agnella Izzo Matic, PhD, CMPP (AIM Biomedical) and was funded by Ferring Pharmaceuticals. The authors thank Iman Mohammadi, PhD, for statistical analysis contributions at Avalere Health.

\section{REFERENCES}

1. Ma GK, Brensinger CM, Wu Q, Lewis JD. Increasing incidence of multiply recurrent Clostridium difficile infection in the United States. Ann Intern Med. 2017;167(3):152-58.

2. Guh AY, Mu Y, Winston LG, et al. Trends in U.S. burden of Clostridioides difficile infection and outcomes. N Engl J Med. 2020;382(14):1320-30.

3. Magill SS, O'Leary E, Janelle SJ, et al. Changes in prevalence of health careassociated infections in U.S. hospitals. N Engl J Med. 2018;379(18):1732-44.

4. Ramai D, Dang-Ho KP, Lewis C, et al. Clostridioides difficile infection in U.S. hospitals: A national inpatient sample study. Int J Color Dis. 2020;35(10):1929-35.

5. Donskey CJ. Clostridium difficile in older adults. Infect Dis Clin N Am. 2017;31(4):743-56.
6. Heinrich K, Harnett J, Vietri J, Chambers R, Yu H, Zilberberg M. Impaired quality of life, work, and activities among adults with Clostridium difficile infection: a multinational survey. Dig Dis Sci. 2018;63(11):2864-73.

7. Smits WK, Lyras D, Lacy DB, Wilcox MH, Kuijper EJ. Clostridium difficile infection. Nat Rev Dis Prim. 2016;2:16020.

8. Lessa FC, Mu Y, Bamberg WM, et al. Burden of Clostridium difficile infection in the United States. N Engl J Med. 2015;372(9):825-34.

9. Leong C, Zelenitsky S. Treatment strategies for recurrent Clostridium difficile infection. Can J Hosp Pharm. 2013;66(6):361-68.

10. Rodrigues R, Barber GE, Ananthakrishnan AN. A comprehensive study of costs associated with recurrent Clostridium difficile infection. Infect Control Hosp Epidemiol. 2017;38(2):196-202.

11. Nour Abou Chakra C, Pepin J. Risk factors for recurrence, complications an mortality in Clostridium difficile infection: a systematic review. PLoS One. 2014;9(6):e98400.

12. Lessa FC, Mu Y, Winston LG, et al. Determinants of Clostridium difficile infection incidence across diverse United States geographic locations. Open Forum Infect Dis. 2014;1(2):ofu048.

13. Deshpande A, Pasupuleti V, Thota P, et al. Risk factors for recurrent Clostridium difficile infection: a systematic review and meta-analysis. Infect Control Hosp Epidemiol. 2015;36(4):452-60.

14. Pechal A, Lin K, Allen S, Reveles K. National age group trends in Clostridium difficile infection incidence and health outcomes in United States community hospitals. BMC Infect Dis. 2016;16(1):682.

15. Balsells E, Shi T, Leese C, et al. Global burden of Clostridium difficile infections: a systematic review and meta-analysis. J Glob Health. 2019;9(1):010407.
16. Garey KW, Sethi S, Yadav Y, DuPont HL. Meta-analysis to assess risk factors for recurrent Clostridium difficile infection. J Hosp Infect. 2008;70(4):298-304.

17. Research Data Assistance Center. Strengths and limitations of CMS administrative data in research. January 10, 2018. Accessed February 23, 2021. https://www.resdac.org/articles/ strengths-and-limitations-cms-administrative-data-research

18. Jacobson G, Freed M, Damico A, Neuman T. A dozen facts about Medicare Advantage in 2019. 2019. Accessed February 23, 2021. http://files.kff.org/ attachment/Data-Note-A-Dozen-FactsAbout-Medicare-Advantage-in-2019

19. Medicare.gov. What Part A covers. 2021. Accessed February 23, 2021. https:// www.medicare.gov/what-medicarecovers/what-part-a-covers

20. Medicare.gov. What Part B covers. 2021. Accessed February 23, 2021. https:// www.medicare.gov/what-medicarecovers/what-part-b-covers

21. Medicare.gov. How to get prescription drug coverage. 2021. Accessed February 23, 2021. https://www. medicare.gov/drug-coverage-part-d/ how-to-get-prescription-drug-coverage

22. Centers for Disease Control and Prevention. Clostridioides difficile infection. November 13, 2019. Accessed February 23, 2021. https://www.cdc.gov/ hai/organisms/cdiff/cdiff_infect.html

23. U.S. Bureau of Labor Statistics. Measuring price change in the CPI: medical care. November 25, 2020. Accessed February 23, 2021. https://www.bls.gov/ cpi/factsheets/medical-care.htm

24. Feuerstadt P, Stong L, Dahdal DN, Sacks NC, Lang K, Nelson WW. Healthcare resource utilization and direct medical costs associated with index and recurrent Clostridioides difficile infection: a real-world data analysis. J Med Econ. 2020;23(6):603-09.

25 . Kelly CP. Can we identify patients at high risk of recurrent Clostridium difficile infection? Clin Microbiol Infect. 2012;18(Suppl 6):21-27. 
26. Zilberberg MD, Shorr AF, Wang L, Baser O, Yu H. Development and validation of a risk score for Clostridium difficile infection in Medicare beneficiaries: a population-based cohort study. J Am Geriatr Soc. 2016;64(8):1690-95.

27. Zacharioudakis IM, Zervou FN, Shehadeh F, Mylona EK, Mylonakis E. Association of community factors with hospital-onset Clostridioides (Clostridium) difficile infection: a population based U.S.-wide study. EClin Med. 2019;8:12-19.

28. Eze P, Balsells E, Kyaw MH, Nair H. Risk factors for Clostridium difficile infections: an overview of the evidence base and challenges in data synthesis. J Glob Health. 2017;7(1):010417.

29. Furuya-Kanamori L, Stone JC, Clark J, et al. Comorbidities, exposure to medications, and the risk of community-acquired clostridium difficile infection: a systematic review and meta-analysis. Infect Control Hosp Epidemiol. 2015;36(2):132-41.

30. Zilberberg MD, Nathanson BH, Marcella S, Hawkshead JJ 3rd, Shorr AF. Hospital readmission with Clostridium difficile infection as a secondary diagnosis is associated with worsened outcomes and greater revenue loss relative to principal diagnosis: a retrospective cohort study. Medicine (Baltimore). 2018;97(36):e12212.
31. Marra AR, Perencevich EN, Nelson RE, et al. Incidence and outcomes associated with Clostridium difficile infections: a systematic review and meta-analysis. JAMA Netw Open. 2020;3(1):e1917597.

32. Collins CE, Ayturk MD, Flahive JM, Emhoff TA, Anderson FA, Santry HP. Epidemiology and outcomes of community-acquired clostridium difficile infections in Medicare beneficiaries. J Am Coll Surg. 2014;218(6):1141-47.e1.

33. Centers for Medicare and Medicaid Services. Post-acute care and hospice provider data 2017. December 3, 2020. Accessed February 23, 2021. https://www. cms.gov/Research-Statistics-Data-andSystems/Statistics-Trends-and-Reports/ Medicare-Provider-Charge-Data/PAC2017

34. Hoffman GJ, Min LC, Liu H, Marciniak DJ, Mody L. Role of post-acute care in readmissions for preexisting healthcare-associated infections. J Am Geriatr Soc. 2020;68(2):370-78.

35. Chopra T, Goldstein EJC. Clostridium difficile infection in long-term care facilities: a call to action for antimicrobial stewardship. Clin Infect Dis. 2015;60 (Suppl 2):S72-76.

36. Rodriguez C, Korsak N, Taminiau B, et al. Clostridium difficile infection in elderly nursing home residents. Anaerobe. 2014;30:184-87.
37. Shah DN, Aitken SL, Barragan LF, et al. Economic burden of primary compared with recurrent Clostridium difficile infection in hospitalized patients: a prospective cohort study. J Hosp Infect. 2016;93(3):286-89.

38. Khanna S, Gupta A, Baddour LM, Pardi DS. Epidemiology, outcomes, and predictors of mortality in hospitalized adults with Clostridium difficile infection. Intern Emerg Med. 2016;11(5):657-65.

39. van Baal P, Morton A, Meltzer D, Brouwer W. Future unrelated medical costs need to be considered in cost effectiveness analysis. Eur J Health Econ. 2019;20(1):1-5.

40. Rappange DR, van Baal PHM, van Exel NJA, Feenstra TL, Rutten FFH, Brouwer WBF. Unrelated medical costs in life-years gained: should they be included in economic evaluations of healthcare interventions? Pharmacoeconomics. 2008;26(10):815-30. 